\title{
1-SS-15 Student Session
}

\section{Enterococcus faecalis extracts FK-23 modulates Propionibacterium acnes- induced lipogenesis in human sebocytes SZ-95 cells}

\author{
Kotone Tanigawa ${ }^{1}$, Kei Ishida ${ }^{1}$, Kousuke Tanaka ${ }^{1}$, China Natsume ${ }^{1}$, Riki Hirashima ${ }^{1}$, \\ Takashi Fujita ${ }^{1}$
}

\section{${ }^{1}$ Molecular Toxicology Lab., Ritsumaikan Univ.}

It is generally accepted that Propionibacterium acnes (P. Acne) is involved in the development of acne, while the mechanisms of sebaceous lipogenesis and its control is unclear. Enterococcus faecalis FK-23 had shown to promote an anti-inflammatory action in several animal models. In the current study, we examined whether FK-23 modulate lipogenesis in sebocytes. FK-23 stimulated lipogenesis, while inhibited them in the presence of P. Acne. FK-23 acutely inhibited acetyl-CoA carboxylase (ACC) phosphorylation levels, while stimulated them with P. Acne. FK-23 stimulated PPAR $\gamma$ expression and activity, while inhibited them with P. Acne as pioglitazone did. These combined evidences demonstrated that the dual action by FK-23 on lipogenesis should reflect differentiation machinery prior to $\operatorname{PPAR} \gamma$, leading to producing adequate levels of sebum. 